\title{
HEIGHT ACCURACY BASED ON DIFFERENT RTK GPS METHOD FOR ULTRA- LIGHT AIRCRAFT IMAGES
}

\author{
K.N. Tahar \\ Centre of Studies for Surveying Science and Geomatics, Faculty of Architecture, Planning and Surveying, Universiti Teknologi \\ MARA, 40450 Shah Alam, Selangor Darul Ehsan, MALAYSIA - khairul0127@ salam.uitm.edu.my
}

\author{
Commission VI, WG VI/4
}

KEY WORDS: Aerial images, positioning, image processing, accuracy

\begin{abstract}
:
Height accuracy is one of the important elements in surveying work especially for control point's establishment which requires an accurate measurement. There are many methods can be used to acquire height value such as tacheometry, leveling and Global Positioning System (GPS). This study has investigated the effect on height accuracy based on different observations which are single based and network based GPS methods. The GPS network is acquired from the local network namely Iskandar network. This network has been setup to provide real-time correction data to rover GPS station while the single network is based on the known GPS station. Nine ground control points were established evenly at the study area. Each ground control points were observed about two and ten minutes. It was found that, the height accuracy give the different result for each observation.
\end{abstract}

\section{INTRODUCTION}

Ultra-light aircraft has been widely used for small scale mapping around the globe. The fully autonomous flight mission helps operator to capture the image at the area of interest autonomously. The ultra-light aircraft were also used in many applications such as historical documentation, stream mapping, landslide mapping, topographical mapping and disaster management (Shahbazi, Theau, \& Menard, 2014; James, et al., 2010; Lee, et al., 2008). The ultra-light aircraft image needs an accurate ground control point in order to achieve the high accuracy products (Shahbazi, et al., 2014). The establishment of ground control point can be done using several instruments such as global positioning system (GPS) and total station. The GPS is quite a popular instrument to obtain the three dimensional coordinates fast and efficient. The total station requires an extra skill to transfer the coordinates from one station to another station. The total station requires a lot of time to transfer the coordinates value at the large area compared to the GPS.

Kung, et al. (2011) has presented the automatic workflow using ultra-light aircraft based on the orientation of the images. The robust system is used to investigate the accuracy of different ground resolutions based on images (Sevcik \& Oh, 2009). This author mentioned that the accuracy achieved about 0.05 to 0.2 meter including GCPs while $2-8 \mathrm{~m}$ with no manual intervention variant. The result of orthomosaic is not well textured at the depth feature. The author also concludes that the resolution and GCP accuracy give an impact to the orthomosaic accuracy. Li (2011) assessed the new development technology namely low altitude UAV for production of large scale topographic map. This study proved that the low altitude is feasible for topographic map. The landslide incident assessment at the area of interest is using 0.3 meter image resolution to determine the sediment of alluvial debris. The author also suggests installing the IMU/DGPS to reduce the ground control point establishment at the field site. The distribution of ground control points must be well distributed to preserve the mapping accuracy. The proper planning by the user will increase the accuracy of the end products.

The GPS observation has many methods such as static, rapid static and real time kinematic observation. One the factor that differentiates these methods is the observation time. The static observation requires about an hour or more, rapid static requires about fifteen minutes to half an hour while real time kinematic requires about 2 minutes observation time. The instruments for static and rapid static are more alike while real time kinematic more like mobile device and easy to carry on the site (Zarzoura, et al., 2013). The accuracy of static method is the most accurate which can achieve about millimeter accuracy level. Rapid static method and real time kinematics can achieve about centimeter to sub centimeter. Static and rapid static methods usually apply for post processing where it used the data from the GPS monument network. Real time kinematic methods can be divided into two networks namely single based and network based. The single based observation only requires one known GPS station to sent the correction to the rover station while network based observation requires multi network known as GPS station to sent the data correction to the rover station. In this study, both observations were used to investigate the effect of the ground control point established from the single based and network based.

\section{MATERIAL AND METHODS}

This study concentrates in the new development area which consist different types of earth surfaces. This study focuses on the two different real time kinematics GPS methods namely single based and network based. In this study, the research methodology is divided into four phases specifically image acquisition, GCP establishment, image processing and analysis. The image acquisition solely obtained from ultra-light aircraft at specific altitude. The preparation of ultra-light aircraft also includes in this phase. The flight mission has been conducted autonomously throughout the study area. The operator is responsible during the flight mission especially during launching and landing. The ground station crew is responsible 
to view or monitor the attitude of ultra-light aircraft during flight mission.

The GCP establishment has been carried out after the flight mission. The selection of GCP is based on the specific characteristics such as location, identification, control point distribution and types of objects selected. The location must be in proper geometry location to accurately reference at the study area. The identification of GCP is most critical due to the resolution of the images. The GCP must be in well distributed and the absolute geometric which minimum has four points. The types of object selected can be identified as the natural or human made object and it must be clear on the image acquired. The image processing requires the photogrammetric software which covers the basic orientations in order to produce the accurate photogrammetric products.

The Photomod software has been used to process the UAV images. Photomod is a Russian product and is known to be one of the most powerful software in photogrammetric image processing. This software is capable to support large number of images in one time especially for large area. The basic orientations include interior orientation, relative orientation and absolute orientation. After went though all orientations, aerial triangulation will be performed to calculate the unknown points on the image measurements. The image processing also covers different GCP configurations during image processing.

In photogrammetric software, tie point measurement can be carried out for each overlapped images. In the Photomod software, there are facilities to measure an accurate location of tie points based on Von Grubber theory. Six box appeares at each model and user can use this box to measure the tie point for each model. Checkpoints (CP) are established to assess the accuracy of photogrammetric results. CP measurement can be done using the point measurement menu by changing the type of point into check. User needs to identify the natural or manmade features which have potential to be a CP. All CPs are established using RTK-GPS. During CP measurement, user needs to make sure that the objects on the image are the same as the real object in the site area. The GCP also has the same concept with the $\mathrm{CP}$ except control points require the user to register the image coordinate into local coordinate system. In contrast, $\mathrm{CP}$ are used to check the photogrammetric results. GCPs are established by using RTK-GPS. 9 GCPs were established evenly at the study area.

UAV raw images were downloaded into a computer after a flight mission is completed. Each image was saved as jpeg file. The quality of images was checked before they were used in the processing stage. Some of the images might have some quality problem such as blurring image and color balancing error which was caused during flight mission. These problems usually arisen from the attitude of the UAV during flight. If the quality of all images are very bad, another flight mission might need to be done. However, in this study, all acquired images were in good quality and they were being proceeded for the photogrammetric processing. As usual, all acquired images need to go through all photogrammetric operation such as interior orientation, exterior orientation, aerial triangulation and bundle adjustment.

As mentioned before, there are six GCP configurations i.e. 4, 5, $6,7,8,9$ GCPs in this study. The results obtained after image processing such as digital elevation model and orthophoto. In analysis phase, the error for each RTK GPS methods were analyzed to determine the effect of error based on different number of GCP configurations. The research methodology for this study describes in Figure 1.

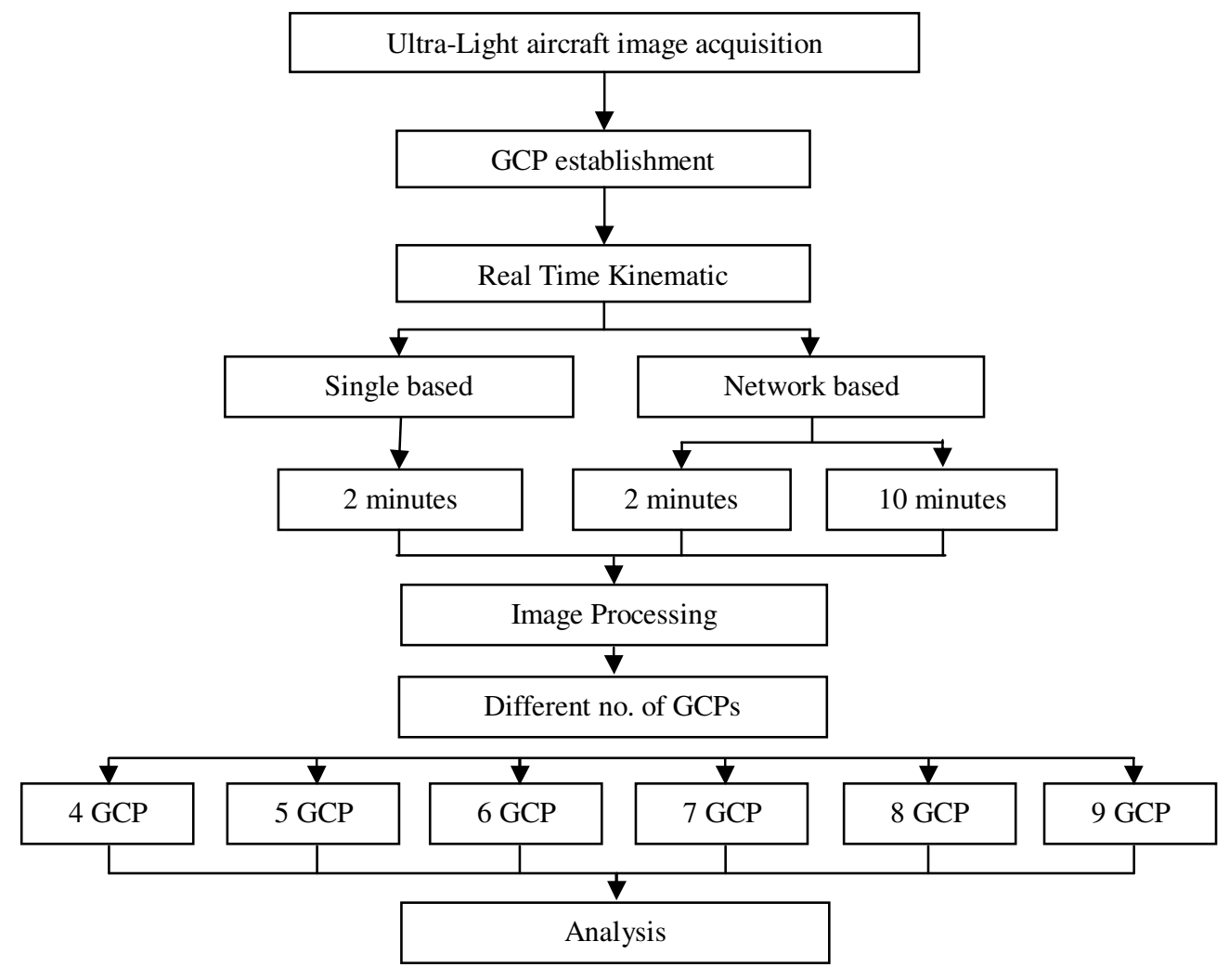

Figure 1. Research Methodology 


\section{RESULTS AND ANALYSIS}

CropCam UAV was employed to acquire aerial images from 320 meters above the surface level. Two primary results were produced in this study; DEM and digital orthophoto. DEM and digital orthophoto were generated after they went through all photogrammetric processes. DEM is generally based on the elevation value while digital orthophoto consists of planimetric position of easting and northing coordinates. The final digital orthophoto can be obtained after mosaic operation using individual orthoimages for each model in the photogrammetric block.

GCPs that were established using RTK-GPS from Iskandar Network (2 minutes), RTK-GPS from a known GPS station (2 minutes) and RTK-GPS from known GPS station (10 minutes) were used as GCP in photogrammetric image processing. Six GCP configurations were applied in this processing namely 4GCP, 5GCP, 6GCP, 7GCP, 8GCP and 9GCP.

Cropcam UAV was utilized for two different experiments based on different RTK GPS data and different GCP configurations. This section discusses on each results and analysis that is obtained from the specific experiment. A discussion on elevation error studies the distribution of $\mathrm{Z}$ coordinate error at the study area. The Cropcam UAV produces 18 photogrammetric results based on different RTK GPS data and different GCP configurations during photogrammetric image processing. The calculation of elevation error was measured based on different actual $\mathrm{Z}$ coordinate and measured coordinates.

\subsection{RTK GPS Iskandar Network (2 minutes)}

There were several checkpoints used to study the distribution of elevation error at the study area. The elevation error went through the interpolation process and it showed the results in graphic view. In this study, the distribution of elevation errors were overlaid with orthophoto using ArcGIS software. Figure 2 illustrates the elevation error for RTK GPS Iskandar Network with two minutes observation using different GCP configurations during photogrammetric image processing.

Based on Figure 2, it found that the highest elevation error focuses at the elevated area while the lowest error locates at the flat area. The range error for each GCP configurations illustrates in Figure 3. Based on Figure 3, it shows that the range error for $4 \mathrm{GCP}$ quite dissimilar with another GCP configurations. The lowest range error records at five to seven GCP. Overall the graph shows the slight growth based on the increment number of GCPs.
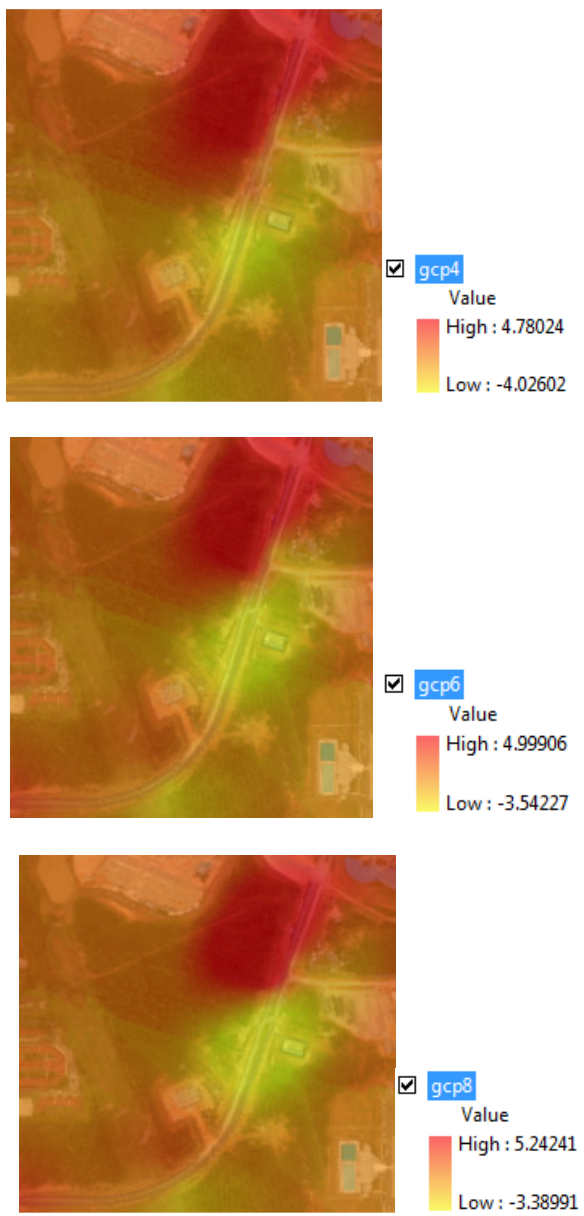
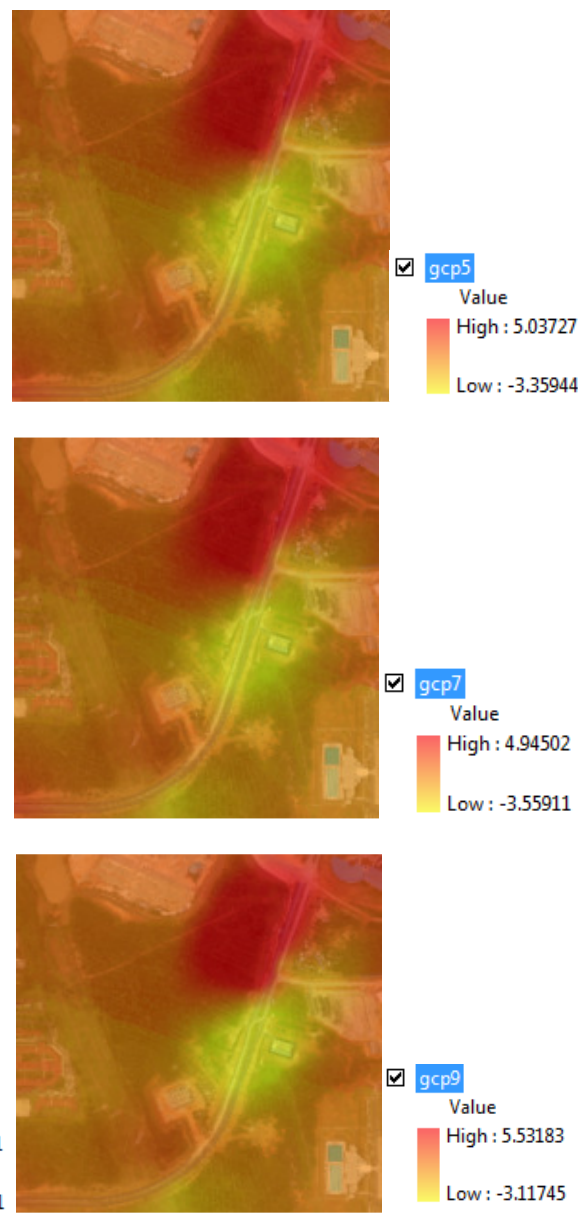

Figure 2. Results on Elevation Error for RTK GPS Iskandar Network (2 minutes) based on different GCP configurations. 


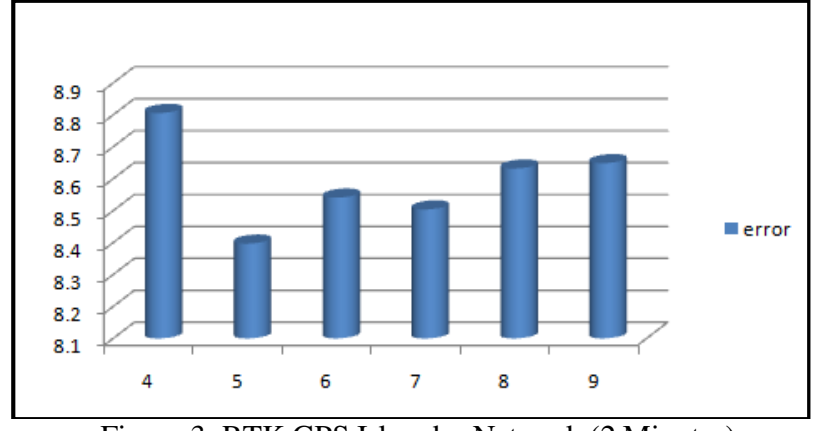

Figure 3. RTK GPS Iskandar Network (2 Minutes)
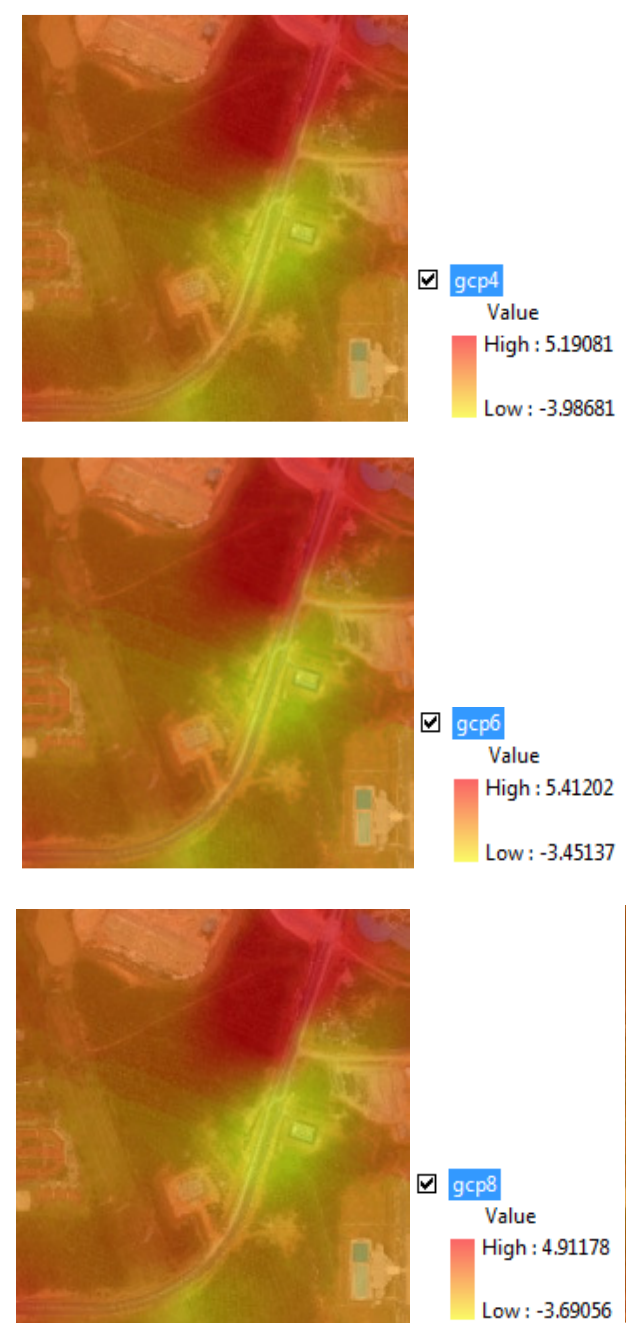

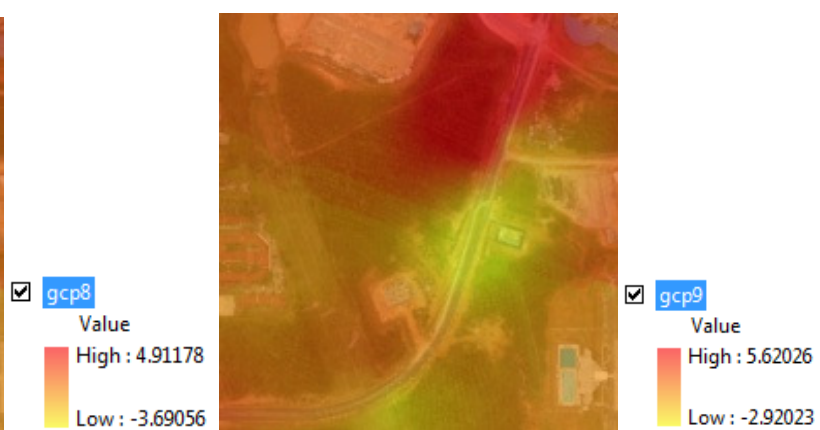

\subsection{RTK GPS from Known GPS Station (2 minutes)}

Figure 4 illustrates the elevation error for RTK GPS from a known GPS Station with two minutes observation using different GCP configurations during photogrammetric image processing. The results for RTK GPS from known GPS station are almost same with the RTK GPS Iskandar Network. It also shows that the huge error concentrates at the elevated area. The range for each GCP configurations describes in Figure 5. Based on Figure 5, it also shows that the range error for 4 GCP record the highest compared to other GCP configurations. The range error for other GCP configurations shows slightly different which about 0.1 to 0.4 meter. The normal distribution graph illustrates for five to nine GCP configurations
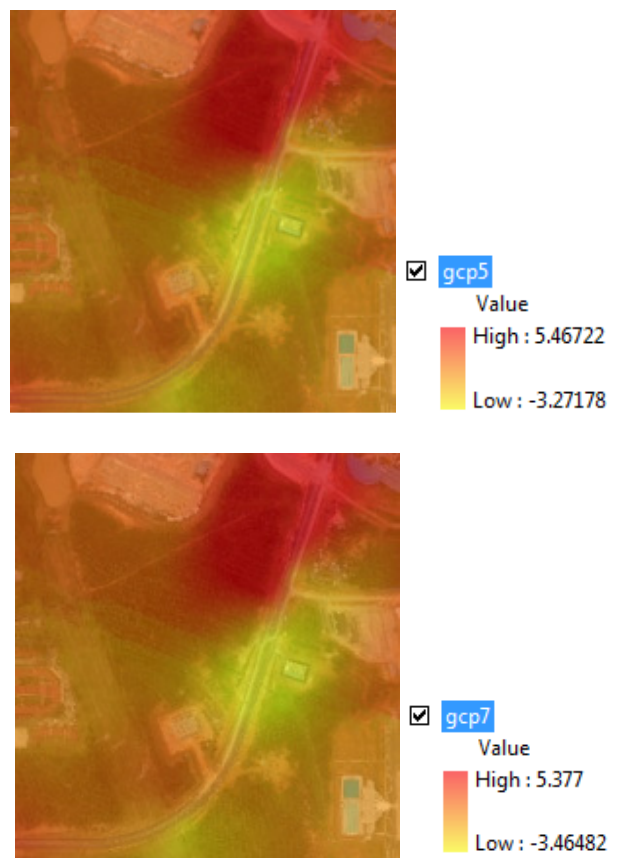

Figure 4. Results on Elevation Error for RTK GPS from Known GPS Station (2 minutes) based on different GCP configurations. 


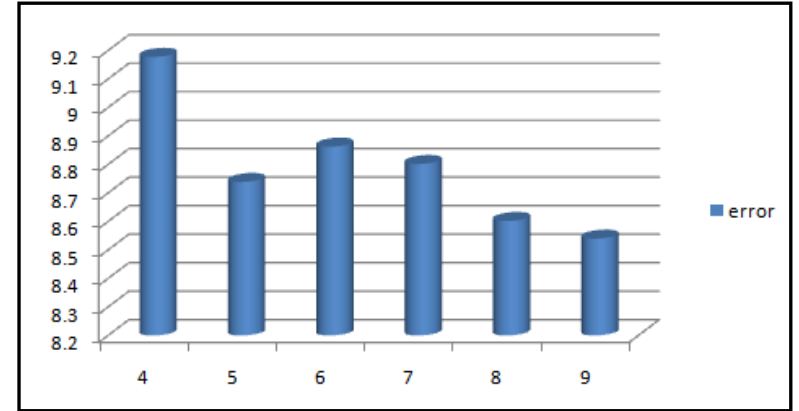

Figure 5. RTK GPS from known GPS Station (2 minutes)

\subsection{RTK GPS from Known GPS Station (10 minutes)}

Figure 6 illustrates the elevation error for RTK GPS from a known GPS Station with ten minutes observation using different GCP configurations during photogrammetric image processing. The elevation error describes the highest error also at the elevated area while lowest error at the flat error. The range error for RTK GPS from known GPS station describes in Figure 7.

Based on Figure 7, it can be seen that the large range records for seven to nine GCP configurations. The lowest range error records at the four to six GCP configurations. The range errors for four to six GCP configurations are almost the same.
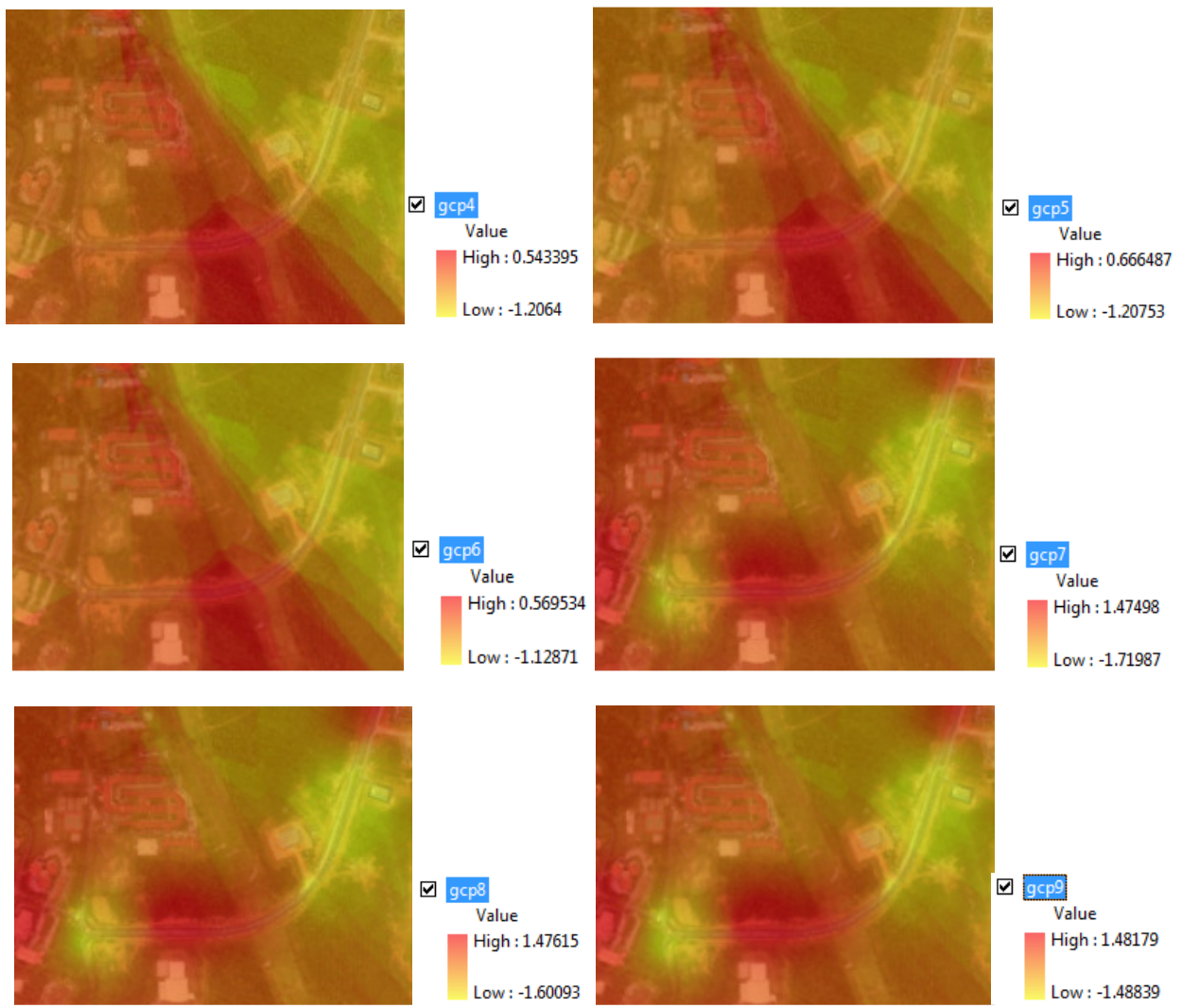

Figure 6. Results on Elevation Error for RTK GPS from Known GPS Station (10 minutes) based on different GCP configurations.

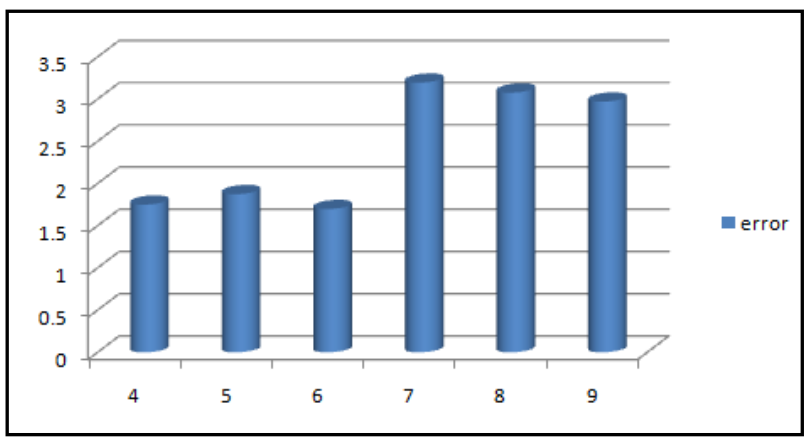

Figure 7. RTK GPS from Known GPS Station (10 minutes)

\section{CONCLUSIONS}

The ultra-light aircraft is very useful for the small scale mapping especially involves the small area. This study investigates the effect on height accuracy based on different GPS observations methods which are single and network based. In addition, the analysis also focuses on the effect of different GCP configurations on the height accuracy. It can conclude that, the network based gave the better result compared to the single based GPS method. However, the observation time also play an important role in determination of height positioning. The result showed that ten minutes observation time could increase the height accuracy at the study area. In future, the extension study on error propagation could be applied to increase the height accuracy. 


\section{ACKNOWLEDGEMENTS}

Faculty of Architecture, Planning and Surveying Universiti Teknologi MARA (UiTM), Research Management Institute (RMi) and Ministry of Higher Education (MOHE) are greatly acknowledged because providing the fund RAGS 600RMI/RAGS 5/3 (241/2014), RAGS/1/2014/TK09/UITM/3 to enable this study is carried out. The authors would also like to thanks people who directly or indirectly involved during this study is carried out.

\section{REFERENCES}

James, N., Niethammer, U., \& Traveletti, J., 2010. Topographic Reconstruction of Landslide Surfaces Using Images from an Unmanned Aerial Vehicle. ISPRS Commission V Mid Term Symposium. Newcastle, UK.

Kung, O., Strecha, C., Beyeler, A., Zufferey, J., Floreano, D., Fua, P., 2011. The accuracy of automatic photogrammetric techniques on ultra-light UAV imagery. Conference unmanned aerial vehicle geomatics.

Lee, C., Jones, S., Bellman, C., \& Buxton, L., 2008. DEM Creation of Snow Covered Surface Using Digital Aerial
Photography. International Archives of the Photogrammetry, Remote Sensing and Spatial Information Sciences. XXXVII, PartB8. Beijing, China.

Li, S. H., 2011. The use of low altitude unmanned aerial vehicle system in the measurement of large scale topographic maps. Kunming, China: Yunnan basic surveying and mapping technology center.

Sevcik, K., \& Oh, P., 2009. Testing Unmanned Aerial Vehicle Missions in Scaled Environment. Journal of Intelligent Robot Systems , pp. 297-305.

Shahbazi, M., Theau, J., \& Menard, P., 2014. Recent application of unmanned aerial imagery in natural resouce management. GIScience \& Remote Sensing , pp. 339-365.

Zarzoura, F., Ehigiator-Irughe, R., \& Mazurov, B., 2013. Inverstigating accuracy enhancement of global navigation satellite system. British journal of earth sciences research , pp. $1-9$. 